EESTI NSV TEADUSTE AKADEEMIA TOIMETISED. XV KÖIDE FUUSIKA-MATEMAATIKA- JA TEHNIKATEADUSTE SEERIA. 1966, NR. 3

НЗВЕСТИЯ АКАДЕМНИ НАУК ЭСТОНСКОИ ССР. ТОМ ХV СЕРИЯ ФИЗИКО-МАТЕМАТИЧЕСКИХХ И ТЕХНИЧЕСКИХ НАУК. 1966, № 3

A. САКОВИЧ, А. МАМСУРОВ, А. НЫВА

\title{
АНАЛИЗ ГАРМОНИЧЕСКОГО СОСТАВА КРИВОЙ ВЫПРЯМЛЕННОГО НАПРЯЖЕНИЯ В УПРАВЛЯЕМОЙ ТРЕХФАЗНОЙ МОСТОВОЙ СХЕМЕ
}

В некоторых преобразователях, питающих нагрузку постоянного тока, может быть полезно использована только гладкая составляющая выходного напряжения, в то время жак все остальные составляющие представляют собой помехи, которые должны быть исключены. Детальный анализ характера этих помех и выявление влияющих на них факторов является основой правильного расчета фильтрующих устройств.

Помехи в кривой выходного напряжения могут быть разделены на следующие группы:

а) пульсации выпрямлснного напряжения с частотсй $\geqslant m f_{1}$ ( $m-$ число коммутаций в схеме, $f_{1}$ - частота питающего напряжения) и

б) пульсация выпрямленного напряжения с частотой $\geqslant \frac{m}{m_{1}} f_{1}$, вызываемые амплитудной и фазовой несимметрией напряжений питающей сети.

\section{Пульсации, вызываемые коммутацией вентилей}

Расчет пульсаций, вызываемых коммутацией вентилей, производится методом гармонического анализа кривой выпрямленного напряжения.

Для вычисления полной гармонической $n$-го порядка могут быть ислользованы известные формулы [1]. При $n=k m$, где $m$ - число коммутаций в схеме за время $T$,

$$
\begin{gathered}
E_{d n m}^{\prime}=\frac{E_{d 0}}{2} \cos \pi m\left[\frac{\sin (k m+1)(\alpha+\gamma)+\sin (k m+1) \alpha}{k m+1}-\right. \\
\left.-\frac{\sin (k m-1)(\alpha+\gamma)+\sin (k m-1) \alpha}{(k m-1)}\right] \\
E_{d n m}^{\prime \prime}=\frac{E_{d 0}}{2} \cos \pi m\left[\frac{\cos (k m+1)(\alpha+\gamma)+\cos (k m+1) \alpha}{(k m+1)}-\right. \\
\left.-\frac{\cos (k m-1)(\alpha+\gamma)+\cos (k m-1) \alpha}{(k m-1)}\right] \\
E_{d n m}=\sqrt{\frac{E_{d n m}^{\prime 2}+E_{d n m}^{\prime \prime 2}}{2}}
\end{gathered}
$$

где $E_{d n m}^{\prime}$ - амплитуда члена синусного ряда;

$E_{d n m}^{\prime \prime}$ - тө же косинусного;

$E_{d n m}-$ амплитуда полной гармонической. 
Входящие в выражения $(1,2)$ величины $\alpha$ и $\gamma$ при регулировании тока в нагрузке с омическим сопротивлением $R_{d}$, изменяющимся в пределах от $R^{\prime}$ до $R^{\prime \prime}$, могут быть вычислены следующим образом:

$$
I_{d}=\frac{U_{d}}{R_{d}}=\frac{1}{R_{d}} \cos \left(\alpha+\frac{\gamma}{2}\right)=\frac{\sqrt{2}}{R_{d}} E_{2} \sin \frac{\pi}{m} \cos \left(\alpha+\frac{\gamma}{2}\right)
$$

нЛи

$$
I_{d}=\frac{\sqrt{2}}{X_{a}} E_{2} \sin \frac{\pi}{m}[\cos \alpha-\cos (\alpha+\gamma)]
$$

откуда

$$
\frac{\cos \alpha-\cos (\alpha+\gamma)}{\cos \left(\alpha+\frac{\nu}{2}\right)}=\frac{X_{a}}{R_{d}}
$$

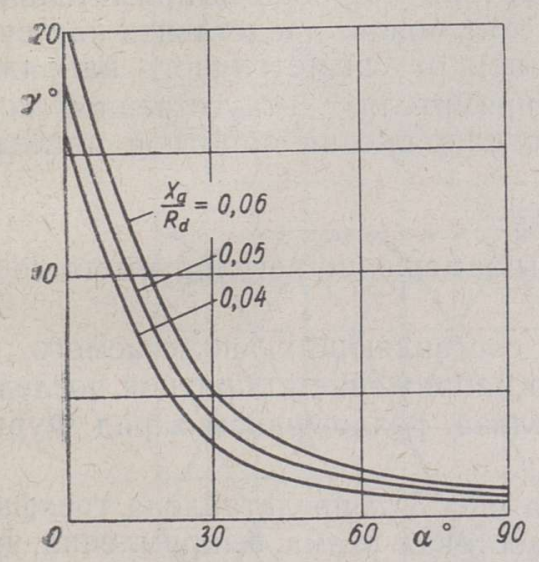

Pнс. 1. График $\gamma=\dot{\varphi}\left(x_{a} / R_{d}, \alpha\right)$

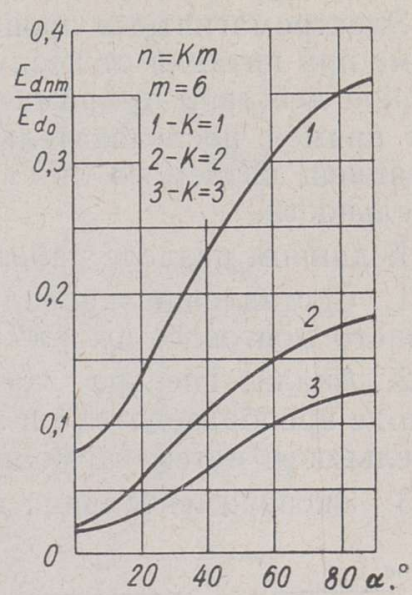

Рис. 2. Гармонический состав кривой выпрямленного напряженгя при различных углах регулирования $\alpha$.

Из уравнения (6) могут быть вычислены численные значения

$$
\gamma=\varphi\left(X_{a} / R_{d} ; \alpha\right)
$$

Эта зависимость построена на рис. 1 для $X_{a} / R_{d}=4 \cdot 10^{-2} \div 6 \cdot 10^{-2}$ и для угла $\alpha$, изменяющегося в пределах от 0 до $\pi / 2$.

На основании полученных данных может быть определен гармонический состав кривой выпрямленного напряжения при различных углах регулирования. Полученные результаты приведены на рис. 2.

\section{Пульсации в кривой выпрямленного напряжения, вызванные несимметрией питающей сети}

Любую несимметричную трехфазную систему можно представить как сумму напряжений прямой, обратной и нулевой последовательности. При анализе выпрямительных схем без использования нулевого привода 
питающего трансформатора или при соединении схемных обмоток в треугольник напряжения нулевой последовательности отсутствуют.

Разложение несимметричной трехфазной ненулевой системы на прямую и обратную составляющие может быть выполнено всегда и притом является единственным. Определение величин векторов производится простым геометрическим способом [2]. Системы векторов $\bar{A}_{1} \bar{B}_{1} \bar{C}_{1}$-и $\bar{A}_{2} \bar{B}_{2} \bar{C}_{2}$ могут быть сдвинуты одна относительно другой на любой угол $\varphi$, характеризуются взаимной обратной последовательностью и в сумме даюг несимметричную систему. Несимметричность трехфазной системы $\eta$ определяется отношением величин векторов прямой и обратной последовательности и обычно исчисляется в процентах.

ӘДС обратной последовательности создают на выходе выпрямителя дополнительные пульсации низкой частоты, требующие (при высоких требованиях к сглаживанию выходного напряжения и тока) применения довольно громоздких фильтрующих устройств.

Электромагнитныв процессы в трехфазной мостовой выпрямительной схеме при питании от несимметричной сети можно представить как суммарное действие выпрямителя с питанием от симметричного напряжения прямой последовательности и выпрямителя с йскусственной коммутацией, питаемого от симметричного напряжения обратной последовательности.

В данном разделе работы излагается:

1. Составление эквивалентной схемы замещения регулируемого трехфазного мостового выпрямителя.

2. Анализ спектра гармонических составляющих, получаемого на выходе преобразователя и вызываемого напряжением обратной последовательности путем гармонического анализа, разложением в ряд Фурье.

3. Экспериментальная проверка.

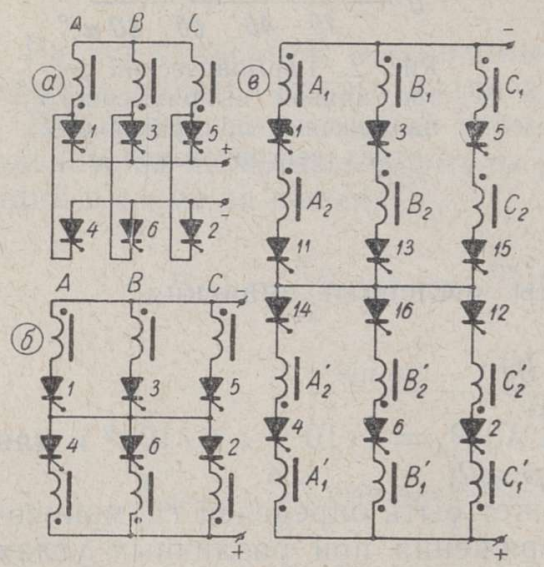

Рис. 3. Преобразование трехфазной мостовой схемы выпрямителя; 8 - принципиальная схема эксперимента.

На рис. 3 , а представлена трехфазная мостовая схема выпрямления, которую можно преобразовать в схему, приведенную на рис. 3 , б. Правомерность такого преобразования очевидна, исходя из возможности разделения обмоток каждой из фаз $A, B$ и $C$ питающего трансформатора на две эквивалентные обмотки с равными ЭДС. В приведенном случае мы имеем трехфазную мостовую схему выпрямления, состоящую из двух нулевых трехфазных схем, на каждую из которых подается симметричное напряжение прямой последовательности.

Включая последовательно в плечо схемы преобразователя обмотки трансформатора, являющиеся источниками ЭДС обратной последовательности, т. е. последовательно $A_{1}$ и $A_{2}$, $B_{1}$ и $C_{2}$ и $C_{1} B_{2}$, и включая коммутирующие вентили $11-16$, получаем схему рис. 3, в, эквивалентную трехфазной мостовой, питаемой от несимметричной сети трехфазного тока $(\varphi=0)$.

Вентили $1-6$ (см. рис. 3,8 ) с питанием от фаз $A_{1}, B_{2}$ и $C_{1}$ являются выпрямителем симметричной сети прямой последовательности, а венти- 
ли $11-16$ с питанием от фаз $A_{2}, B_{2}$ и $C_{2}$ - выпрямителем симметричной сети обратной последовательности с искусственной коммутацией.

На рис. 4 приведено построение кривых низкочастотной модуляции выходного напряжения, т. е. кривых на выходе двух нулевых схем $\boldsymbol{U}_{d o n 1}, U_{d \text { оп2 }}$ и суммарной кривой $\Sigma U_{d \text { оп }}$, вызываемой напряжением обратной последовательности при значении угла регулирования $\alpha=60^{\circ}$ (без учета угла коммутации $\nu$ ).

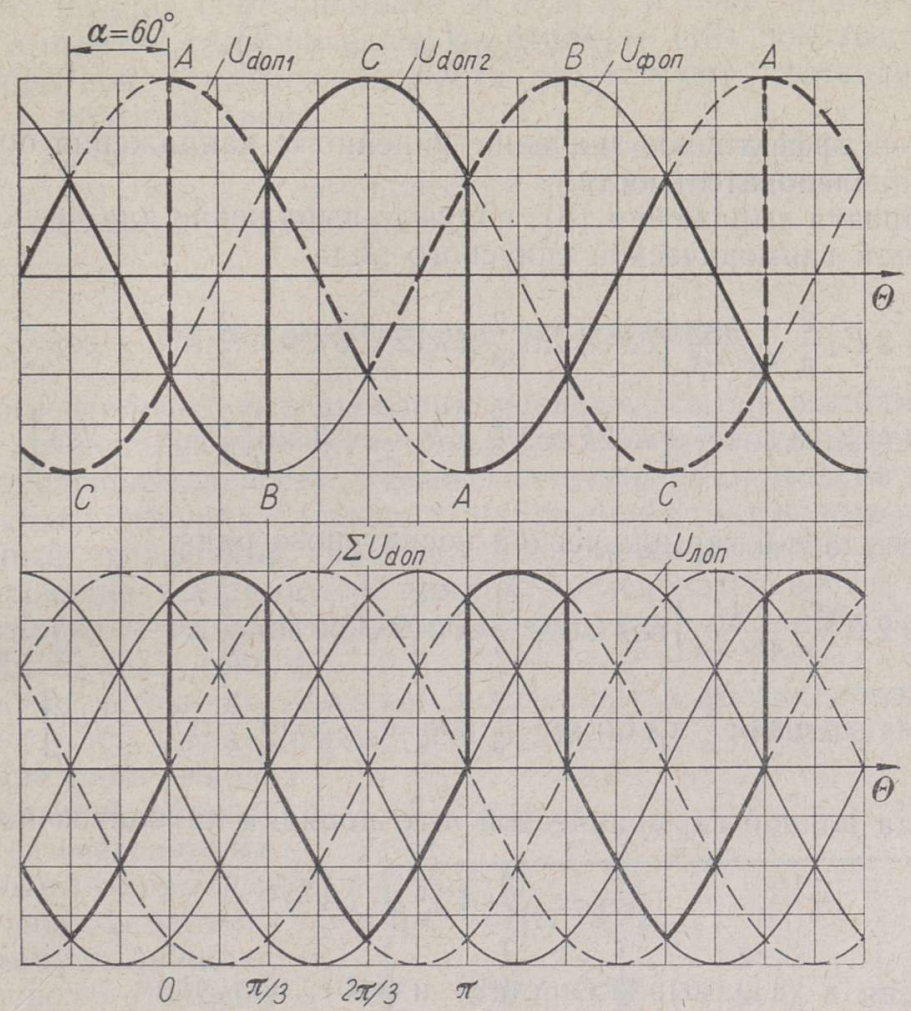

Рис. 4. Построение кривых низкочастотной модуляции выходного напряжения при $\alpha=60^{\circ}$.

Кривая выходного напряжения от несимметричной сети является суммой кривых, образованных напряжениями прямой и обратной последовательности, и каждая из этих кривых имеет свой спектр гармонических составляющих.

Форма кривой выпрямленного напряжения обратной последовательности соответствует, в данном случае, непрерывному току нагрузки.

Значения амплитуд гармонических составляющих можно получить, интегрируя кривую выпрямленного напряжения по периоду повторяемости, который в этом случае разо́ивается на три участка:

$$
\begin{aligned}
& \Delta t_{1} \rightarrow 0<\Theta<\frac{\pi}{3} \\
& \Delta t_{2} \rightarrow \frac{\pi}{3}<\Theta<\frac{2 \pi}{3} \\
& \Delta t_{3} \rightarrow \frac{2 \pi}{3}<\Theta<\pi .
\end{aligned}
$$


Для каждого из участков кривой выпрямленного напряжения записывается в виде

$$
\begin{aligned}
& \sqrt{2} E_{2}^{\prime} \sin (\Theta+\alpha) \\
& \sqrt{2} E_{2}^{\prime} \cos \left(\Theta+\alpha-\frac{\pi}{6}\right) \\
& \sqrt{2} E_{2}^{\prime} \cos \left(\Theta+\alpha+\frac{\pi}{6}\right)
\end{aligned}
$$

где $E_{2}^{\prime}$ - эффективное значение линейного напряжения обратной последовательности.

Преобразуя выражения (8), получим выражение для вычисления амплитуды $n$-й гармонической синусного ряда

$$
\begin{aligned}
A^{\prime}= & \sqrt{2} E_{2}^{\prime} \frac{2}{\pi} \frac{1}{4 k^{2}-1}\left[\cos \alpha \sin \frac{2}{3} \pi k\left(2 \sqrt{3} k \sin \frac{2}{3} \pi k-\cos \frac{2}{3} \pi k+1\right)+\right. \\
& \left.+\sin \alpha \cos \frac{2}{3} \pi k\left(2 k \cos \frac{2}{3} \pi k-\sqrt{3} \sin \frac{2}{3} \pi k-2 k\right)\right]
\end{aligned}
$$

Амплитуда $n$-й гармонической косинусного ряда

$$
\begin{aligned}
A^{\prime \prime}= & \sqrt{2} E_{2}^{1} \frac{2}{\pi} \frac{1}{4 k^{2}-1}\left[\cos \alpha \cos \frac{2}{3} \pi k\left(2 \sqrt{3} k \sin \frac{2}{3} \pi k-\cos \frac{2}{3} \pi k+1\right)-\right. \\
& \left.-\sin \alpha \sin \frac{2}{3} \pi k\left(2 k \cos \frac{2}{3} \pi k-\sqrt{3} \sin \frac{2}{3} \pi k-2 k\right)\right] .
\end{aligned}
$$

Амплитуда полной гармонической $n$-го порядка находится из равенства

$$
A=\sqrt{\left(A^{\prime}\right)^{2}+\left(A^{\prime \prime}\right)^{2}} .
$$

Подставляя в (11) выражение (9) и (10), получаем

$$
A=\sqrt{2} E_{2}^{\prime} \frac{2}{\pi} \frac{1}{4 k^{2}-1}\left(2 \sqrt{3} k \sin \frac{2}{3} \pi k-\cos \frac{2}{3} \pi k+1\right) .
$$

\section{Полагая}

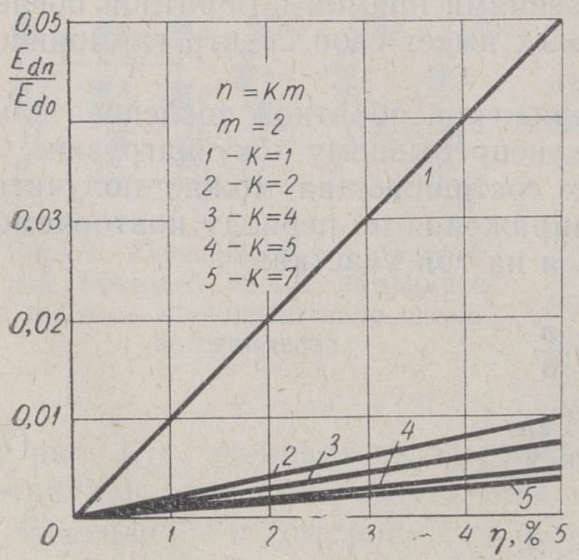

$$
\begin{aligned}
\cos \frac{2}{3} \pi k & =\cos \frac{4}{3} \pi k \\
\left(1-\cos \frac{4}{3} \pi k\right) & =2 \sin ^{2} \frac{2}{3} \pi k \quad \text { и } \\
\sqrt{2} E_{2}^{\prime} & =\frac{2}{3} \eta E d_{0}
\end{aligned}
$$

Рис. 5. Спектр гармонических колебаний, вызываемый напряжением обратной последовательности. 
где $\eta=E_{2}^{\prime} / E_{2}$ - коэффициент несимметрии, получаем окончательное выражение для расчета амплитуд гармонических составляющих, не зависящее от величины угла запаздывания $\alpha$ :

$$
A=\frac{2}{3} \eta E_{d_{0}} \frac{2 \sin \frac{2}{3} \pi k}{4 k^{2}-1}\left(\sin \frac{2}{3} \pi k+\sqrt{3} k\right)
$$

для данного периода повторяемости от 0 до $\pi, n=k m$, при $m=2$.

Расчет амплитуд гармонических по формуле (13) показывает, что в спектре обратной последовательности отсутствуют составляющие со значением $k$, кратным трем.

График, приведенный на рис. 5, характеризует состав и величину дополнительного спектра гармонических выходного напряжения в зависимости от величины несимметрии $\eta$.

\section{Әкспериментальные исследования}

Экспериментальные исследования проводились на выпрямительном устройстве, моделирующем схему рис. 3 , в, с выделением составляющей обратной последовательности. Схема эксперимента приведена на рис. 6.

В качестве источника питающего напряжения был использован многообмоточный трехфазный трансформатор мощностью 25 ква. Напряжения, питающие выпрямитель прямой последовательности, включающий тиристоры $1-6$, моделировались обмотками $W_{1}-W_{6}$ расщепленных фаз $A_{1}, B_{1}$ и $C_{1}$ (рис. 6).

В качестве источника обратной последовательности использовались обмотки $W_{7}-W_{12}$ расщепленных фаз $A_{2}, B_{2}$ и $C_{2}$ с тиристорами $11-16$

Для выделения выход-

ных напряжений, образо-

ванных симметричными составляющими прямой и обратной последовательности, в схему были включены вспомогательные диоды 20-31 и сопротивления $R_{1}-R_{4}$.

Таким образом, в схеме оказываются выделенными на сопротивлениях $R_{1}$ и $R_{4}$ выходные напряжения двух трехфазных нулевых выпрямителей, составляющих в сумме мостовой выпрямитель, питающийся напряжением прямой последовательности.

Кривые выходного напряжения соответствующих выпрямителей обратной последовательности выделены в узлах схемы 4,5 и 3,5 на осциллограммах рис. $7, a, \sigma$ при-

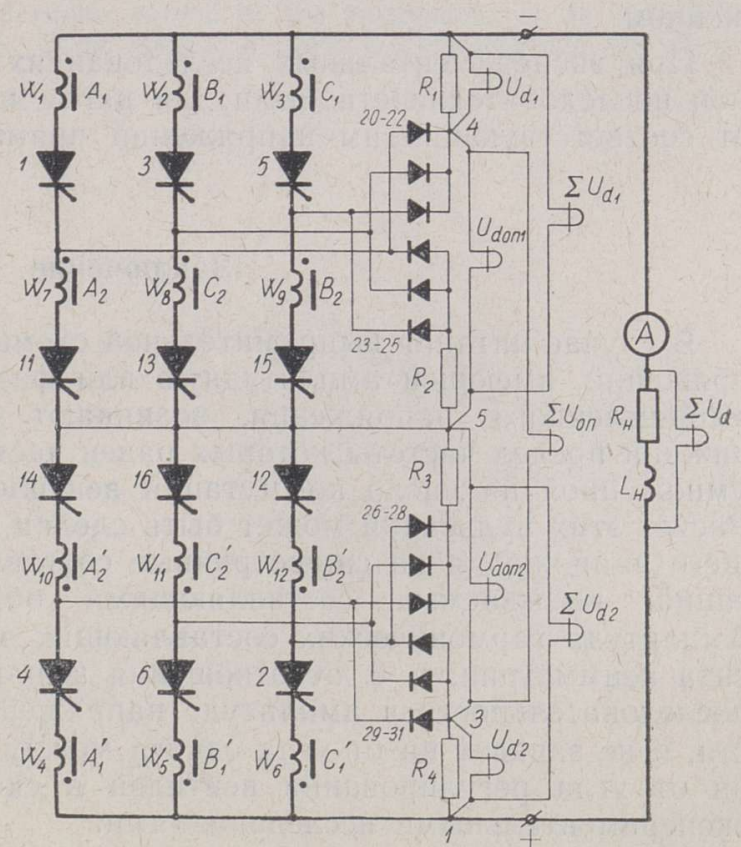

Рис. 6. Схема эксперимента. 


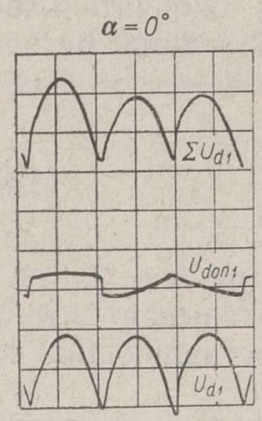

$a$

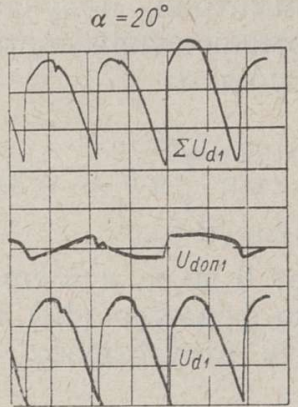

$\sigma$

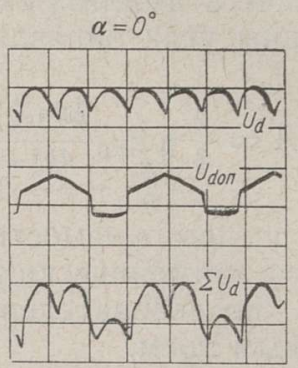

$b$

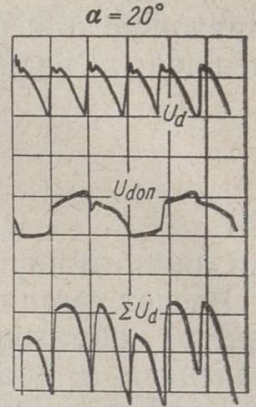

2

Рис. 7. Осциллограммы выходного напряжения нулевой схемы при $\alpha=0 \quad(a)$ и $20^{\circ}(б)$; то же мостовой схемы при $\alpha=0$ (в) и $20^{\circ}$ (2).

ведены кривые, характеризующие работу одной из нулевых схем при $\alpha=0$ и $20^{\circ}$.

Кривые $U_{d 1}$ иллюстрируют работу нулевой схемы, питаемой симметричным напряжением прямой последовательности, кривые $U_{d \circ \pi 1}-$ низкочастотную модуляцию, вызываемую напряжением обратной последовательности, и $\Sigma U_{d 1}$ - суммарную кривую, получаемую на выходе от питания несимметричным напряжением. Осциллограммы на рис. 7 , 8 , г характеризуют работу мостовой схемы при $\alpha=0$ и $20^{\circ}$. На рисунке приведены: кривая выходного напряжения симметричной мостовой схемы $U_{d}$, кривая низкочастотной модуляции $\Sigma U_{d o n}$, являющаяся суммой модуляций $U_{d \circ \text { п }}$ и $U_{d o n 2}$, и $\sum U_{d}-$ суммарная кривая выходного напряжения мостового выпрямителя, питаемого асимметричным напряжением.

При экспериментальных исследованиях фазные напряжения обратной последовательности были для наглядности приняты равными $10 \%$ от соответствующих им напряжений прямой последовательности.

\section{Заключение}

В случае питания выпрямительной схемы от трехфазной системы напряжений, имеющей амплитудную или фазовую асимметрию в кривой выпрямленного напряжения, возникают дополнительные пульсации, нижний предел частоты которых равен частоте питающего напряжения, умноженной на число коммутаций вентилей на период в одной фазе. Расчет этих пульсаций может быть сделан методом разложения питающего напряжения на симметричные составляющие и выделением пульсаций, вызываемых составляющими обратной последовательности. Амплитуда гармонических составляющих зависит только от коэффициента асимметрии, т. е. от отношения амплитуды напряжения обратной последовательности к амплитуде напряжения прямой последовательности, и не зависит ни от угла сдвига между указанными напряжениями, ни от угла регулирования вентилей в схеме. Расчеты подтверждены экспериментальными исследованиями.

Наличие в кривой выпрямленного напряжения гармонических низких частот, обусловленных асимметрией питающих напряжений, делает 
в большинстве случаев неэффективным применение обычных фильтров

с реактивными элементами (при необходимости хорошего сглаживания они получаются чрезмерно громоздкими).

\section{ЛИТЕ Р А Т У Р А}

1. К а ганов И. Л., Электронные и нонные преобразователи, ч. III, М., 1956.

2. К руг К. А., Основы электротехники, т. II, М., 1962.

Ннститут термофизики и электрофизики

Академии наук Эстонской ССР

Всесоюзный электротехнический институт им. В. Н. Ленина

\section{Поступила в редакцию} 11/XI 1965

A. SAKOVITS, A. MAMSUROV, A. NÖVA

\section{KOLMEFAASILISE TUURITAVA SILDALALDAJA VÄLJUNDPINGE HARMOONILINE ANALUUS}

Artiklis on matemaatiliselt avaldatud tüüritava alaldaja väljundis esinevad pulsatsioonipinge harmoonilised komponendid. On tuletatud valem toitepinge asümmeetriast tingitud pulsatsiooni avaldamiseks. Saadud tulemusi on kontrollitud eksperimentaalselt.

A. SAKOVICH, A. MAMSUROV, A. NOVA

\section{ANALYSIS OF HARMONIC VOLTAGES IN THE OUTPUT OF A CONTROLLED THREE-PHASE BRIDGE RECTIFIER}

In this article the harmonic voltages present in the output of rectifier circuits are derived mathematically. A factor of ripple, caused by the assymmetry of the three-phase system, is obtained mathematically. These results of mathematical analysis are checked up experimentally. 